\title{
Critical temperatures in the synthesis of graphene-like materials by thermal exfoliation-reduction of graphite oxide
}

Cristina Botas ${ }^{1}$, Patricia Álvarez ${ }^{1}$, Clara Blanco ${ }^{1}$, Ricardo Santamaría ${ }^{1}$, Marcos Granda $^{1}$, M. Dolores Gutiérrez ${ }^{2}$, Francisco Rodríguez-Reinoso ${ }^{3}$ and Rosa Menéndez ${ }^{1, *}$

${ }^{1}$ Instituto Nacional del Carbón (INCAR-CSIC), Apdo. 73, 33080, Oviedo (Spain)

${ }^{2}$ Departmento de Química Física y Analítica, Universidad de Oviedo, C/ Julián Clavería, $\mathrm{S} / \mathrm{N}, 33006-O v i e d o$ (Spain)

${ }^{3}$ Universidad de Alicante, Apdo 99, E-03080 Alicante (Spain)

\begin{abstract}
We prepared a series of graphene-like materials by thermal exfoliation/reduction of a graphite oxide $(\mathrm{GO})$ at temperatures between $127^{\circ} \mathrm{C}$ and $2400^{\circ} \mathrm{C}$. The extent of the exfoliation and reduction of the GO at different temperatures, as well as the impact on the resultant graphene-like materials (TRGs), were studied through their chemical/structural characterization. The main oxygen loss was observed at $127^{\circ} \mathrm{C}$ during the blasting of the GO, which produced its exfoliation into monolayer functionalized TRG with hydroxyl groups and minor amounts of epoxy and carboxyl groups. Above $600^{\circ} \mathrm{C}$, the reduction continued smoothly, with oxygen and hydrogen loss and the conversion of hybridized carbon atoms from $\mathrm{sp}^{3}$ into $\mathrm{sp}^{2} \cdot 1000^{\circ} \mathrm{C}$ appears to be a critical temperature for the efficiency of the reduction process, as the resulting TRG contained less than $2 \%$ oxygen and $81.5 \% \mathrm{sp}^{2}$-carbon atoms. The materials obtained at $2000^{\circ} \mathrm{C}$ and $2400^{\circ} \mathrm{C}$ were almost oxygen-free and the layers exhibited a dramatic restoration of the pristine graphite structure, as confirmed by the increase in the average size of the $\mathrm{sp}^{2}$-domains. The typical
\end{abstract}

\footnotetext{
* Corresponding author: Fax. + 349852976 62; E-mail: rosmenen@incar.csic.es (Prof. R. Menéndez)
} 
disordered stacking of TRGs increases with temperature, although they can be dispersed yielding monolayers at 127 and $300^{\circ} \mathrm{C}$ and stacks of up to $4-6$ layers above $1000^{\circ} \mathrm{C}$, as determined by AFM.

\section{Introduction}

Graphene and graphene-based materials have attracted a great deal of interest due to their fascinating properties and potential applications [1]. The exfoliation of GO to yield graphene oxide [2] followed by a reduction process offers a simple method to obtain graphene [3]. This method has the advantage of being readily scalable and also has the ability to produce graphene materials with a tuneable amount of oxygen, depending on the characteristics of the parent graphite [4] and the experimental conditions of both oxidation and reduction $[2,5]$. Several processes have been considered for the reduction of graphene oxide. These include: i) chemical reduction with different reagents, hydrazine being the most common [5]; ii) thermal reduction which can be microwave assisted or performed in different atmospheres (argon, vacuum, hydrogen, hydrogen/argon) [6]; iii) electrochemical reduction [7] and iv) a combination of different methods, such as chemical reduction/thermal annealing or thermal reduction/hydrogenation [6]. It is important to note that in the case of the thermal reduction the process includes the liberation of the bound oxygen in the form of $\mathrm{CO}$ and $\mathrm{CO}_{2}$, as oxidation products of carbon, and the reduction of the remaining carbon. In this regard, thermal exfoliation/reduction of GO is an interesting alternative to chemical reduction for the preparation of graphene materials [3] because of: i) its simplicity, since the exfoliation of the GO to graphene oxide and its thermal reduction to graphene occurs all in one step; ii) its sustainability, since it avoids the use of 
environmentally unfriendly chemicals; and iii) its scalability. Moreover, the graphene-like materials obtained by this procedure are electrically conducting, thus overcoming the poor electrical and thermal properties of the parent GO [8] and making possible their use in electrical transport applications [9]. The efficiency with which graphene single sheets can be prepared by the thermal expansion of GO and the quality of the graphene sheets is dependent on the degree of oxidation of the graphite and the conditions of the thermal treatment. One of the requirements is to build up enough pressure during heat treatment stages [10]. Exfoliation takes place when the decomposition rate of the oxygen functional groups of GO exceeds the diffusion rate of the evolved gases, thus yielding pressures that exceed the Van der Waals forces that hold the graphene sheets together. Consequently, it is essential for the success of the process to completely eliminate the intergraphene spacing associated with the native graphite during the oxidation stage and to minimize the detrimental effects of water vapour. Rapid heating of GO obtained from flake graphite to a temperature of $1050{ }^{\circ} \mathrm{C}$ has been used $[9,10]$ to prepare bulk quantities of single functionalized graphene sheets after dispersion by ultrasonication. The study of the thermal expansion mechanism involved suggested that the critical temperature of $550{ }^{\circ} \mathrm{C}$ must be exceeded for exfoliation to occur [10]. The graphene materials obtained by this method have a wrinkled structure, with a $\mathrm{C} / \mathrm{O}$ ratio of $10: 1$, and are electrically conducting. In the as-prepared TRG powder the sheets were highly agglomerated and randomly stacked. No reference was made in these studies to the characteristics of the residual oxygen functional groups. Gao et al. [3] used the density functional theory to study the reaction mechanisms involved in the reduction of graphene oxide with hydrazine and thermal treatment. They described the mechanisms of dehydroxilation, decarbonylation and decarboxylation when using heat treatment and concluded that the oxygen functionalities attached to the interior 
of an aromatic domain on the basal plane are removed more easily, both kinetically and thermodynamically, than those attached to the edges of an aromatic domain. Bagri et al. [11] made use of molecular dynamics simulations supported by FTIR and XPS to study the chemical changes of the oxygen functional groups upon the annealing of the graphene oxide and concluded that the formation of highly stable carbonyl and ether groups (through the transformation of the initial hydroxyl and epoxy groups) hinders the complete reduction of graphene oxide to partially reduced graphene. Nonetheless, further experimental evidence is needed to support their studies. Moreover, to our knowledge, there is no detailed, systematic study on how the experimental conditions of the thermal treatment (heating rate, the use of vacuum or pressure, final temperature) may affect the efficiency of the process and the structure of the final graphene-like material.

The main objective of this work is to study the effect of temperature on the extent of the exfoliation and reduction of $\mathrm{GO}$, as well as its impact on the resultant graphene-like materials. A commercial synthetic graphite powder was oxidized by a modified Hummers' method $[4,12]$ and thermally treated at temperatures ranging from $127^{\circ} \mathrm{C}$ to $2400{ }^{\circ} \mathrm{C}$ in order to: i) determine the optimum temperature for the preparation of graphene-like materials (TRGs) with a minimum oxygen content and a minimum amount of structural defects; ii) obtain graphene-like materials with different degree of functionalization; and iii) study the effect of the temperature on the degree of association of the sheets. Elemental analysis, TPD (temperature programmed desorption), XRD, Raman spectroscopy, XPS, FTIR, SEM, TEM, high resolution TEM and AFM were used to monitor the chemical and structural changes occurring in the TRG produced at each temperature. 


\section{Experimental}

\subsection{Preparation of graphite oxide (GO).}

Commercial synthetic graphite powder supplied by Sigma Aldrich $(<20 \mu \mathrm{m})$ was used as starting material for the preparation of the samples in this study. The ash content of the graphite, as determined by TGA was $1.9 \%$. The carbon content, on an ash-free basis, was $99.5 \%$.

GO was prepared from the commercial graphite powder using a modified Hummers' method $[4,12]$. This method makes use of the Hummers' reagents with additional amounts of $\mathrm{NaNO}_{3}$ and $\mathrm{KMnO}_{4}$. Concentrated $\mathrm{H}_{2} \mathrm{SO}_{4}(360 \mathrm{~mL})$ was added to a mixture of graphite $(7.5 \mathrm{~g})$ and $\mathrm{NaNO}_{3}(7.5 \mathrm{~g})$, and the mixture was cooled down to $0{ }^{\circ} \mathrm{C}$ by means of an ice bath. $\mathrm{KMnO}_{4}(45 \mathrm{~g})$ was added slowly in small doses to keep the reaction temperature below $20{ }^{\circ} \mathrm{C}$. The solution was heated to $35{ }^{\circ} \mathrm{C}$ and stirred for $3 \mathrm{~h}$, at which point $3 \% \mathrm{H}_{2} \mathrm{O}_{2}$ $(1.5 \mathrm{~L})$ was slowly added, giving rise to a pronounced exothermal effect to $98{ }^{\circ} \mathrm{C}$. The reaction mixture was stirred for $30 \mathrm{~min}$ and, finally, the mixture was centrifuged $(3700 \mathrm{rpm}$ for $30 \mathrm{~min}$ ), the supernatant being decanted away. The remaining solid material was then washed with $600 \mathrm{~mL}$ of water and centrifuged again, this process being repeated until the $\mathrm{pH}$ was neutral [4]. When required, GO was dried under vacuum at $40{ }^{\circ} \mathrm{C}$ overnight and stored in the presence of $\mathrm{P}_{2} \mathrm{O}_{5}$ as desiccant.

\subsection{Preparation of graphene-like materials (TRGs).}

The GO was thermally treated in a Thermal Programmed Desorption (TPD) device in order to determine the blasting temperature. The system consists of an electrical furnace with a U-shape quartz glass reactor connected to a mass spectrometer (Omnistar TM-Pheiffer 
Vacuum). Initially, the sample (100 mg) was degassed under a He flow $\left(50 \mathrm{~mL} \mathrm{~min}^{-1}\right)$ at room temperature for $1 \mathrm{~h}$. Then the sample was heated from room temperature to $127^{\circ} \mathrm{C}$ at a heating rate of $5{ }^{\circ} \mathrm{C} \min ^{-1}$. At this temperature blasting occurs as a consequence of the sudden release of gases (see Supporting Information, S.I.). The resultant sample was labelled TRG127.

The temperatures used for the preparation of rest of the samples were selected from the thermogravimetric analysis (TGA/DTG) of the graphite oxide exfoliated by sonication. GO was not used directly for these experiments to avoid the blasting and blowing of the material out of the vessel. Analyses were carried out using a TA SDT 2960 analyzer. $5 \mathrm{mg}$ of sample were placed in a crucible that was then introduced into the thermobalance. The temperature was increased to $1000{ }^{\circ} \mathrm{C}$ at a heating rate of $5^{\circ} \mathrm{C} \mathrm{min}{ }^{-1}$ under a nitrogen flow of $100 \mathrm{~mL} \mathrm{~min}^{-1}$. Based on these results, samples were obtained in a horizontal tube furnace using a ceramic boat with a graphite cover to prevent the blowing of the material. $0.3 \mathrm{~g}$ of $\mathrm{GO}$ was introduced in the furnace and heated at $5^{\circ} \mathrm{C} \mathrm{min}^{-1}$ under an atmosphere of $\mathrm{N}_{2}(100$ $\mathrm{mL} \min ^{-1}$ ) to $300{ }^{\circ} \mathrm{C}, 400{ }^{\circ} \mathrm{C}, 600{ }^{\circ} \mathrm{C}, 700{ }^{\circ} \mathrm{C}, 800{ }^{\circ} \mathrm{C}$ and $1000{ }^{\circ} \mathrm{C}$, the samples being then kept at each of these temperatures for $1 \mathrm{~h}$. Additionally, the sample obtained at $700{ }^{\circ} \mathrm{C}$ was annealed at $2000{ }^{\circ} \mathrm{C}$ and $2400{ }^{\circ} \mathrm{C}$ under an atmosphere of argon $\left(3 \mathrm{~L} \mathrm{~min}^{-1}\right)$ at a heating rate of $5{ }^{\circ} \mathrm{C} \min ^{-1}$ to $800{ }^{\circ} \mathrm{C}$ and then at $10{ }^{\circ} \mathrm{C} \mathrm{min}^{-1}$ to 2000 or $2400{ }^{\circ} \mathrm{C}$, respectively, the samples were kept at this temperature for $1 \mathrm{~h}$. The samples obtained were labelled TRG300, TRG400, TRG600, TRG700, TRG800, TRG1000, TRG2000 and TRG2400, respectively. 
A colloidal suspension of individual TRG sheets in purified water/DMF (1:1) was prepared in $1 \mathrm{~mL}$ batches, and kept under ultrasound for $30 \mathrm{~min}$. Then the suspensions (see S.I.) were filtered over cellulose, and the solids were discarded.

\subsection{Characterization of samples.}

The oxygen content of the samples was determined directly in a LECO-TF-900 furnace coupled to a LECO-CHNS-932 microanalyzer. The analyses were performed using $1 \mathrm{mg}$ of ground sample. The results were quoted from an average of the values of four determinations. In all cases, the experimental error was $<0.5 \%$ of the absolute value. XPS analyses were carried out in a VG-Microtech Mutilab 3000 device. The XPS C1s peak was analyzed using a peak synthesis procedure that employs a combination of Gaussian and Lorentzian functions [13] in order to identify the functional groups and the respective percentages. The binding energy profiles were deconvoluted as follows: undamaged

structures of $\mathrm{Csp}^{2}$-hybridized carbon $(284.5 \mathrm{eV})$, damaged structures or $\mathrm{sp}^{3}$-hybridized carbons $(285.5 \mathrm{eV}), \mathrm{C}-\mathrm{OH}$ groups $(286.5 \mathrm{eV}), \mathrm{O}-\mathrm{C}-\mathrm{O}$ functional groups $(287.7 \mathrm{eV})$ and $\mathrm{C}(\mathrm{O}) \mathrm{OH}$ groups at $288.7 \mathrm{eV})$. FTIR spectra of $\mathrm{GO}$ were recorded at room temperature using an attenuated total reflection (ATR) mode and a Fourier transform infrared spectrometer (FTIR, Nicolet 8700 FTIR, Thermo Scientific) fitted with a DFT (deuterated triglycine sulfate) detector. Solid samples were placed on a single-bound diamond plate. Sixty four scans $\left(4 \mathrm{~cm}^{-1}\right.$ resolution) per sample were recorded between 3700 and $600 \mathrm{~cm}^{-1}$. XRD analysis of powdered samples was performed using a Bruker D8 Advance diffractometer. The radiation frequency employed was the $\mathrm{K} \alpha 1$ line from $\mathrm{Cu}(1.5406 \AA)$, with a power supply of $40 \mathrm{kV}$ and $40 \mathrm{~mA}$. The crystallite size along the c-axis $\left(\mathrm{L}_{\mathrm{c}}\right)$ and the interlaminar distances of sheets were obtained from the (002) reflection of the XRD 
patterns $\left((001)\right.$ reflection in the case of GO), which were recorded at steps of $0.01^{\circ}$ and intervals of $6 \mathrm{~s}$ per step, using the Scherrer equation. SEM images were obtained using a field emission gun scanning electron microscope (QUANTAN FEG 650, FEI) operating at $5 \mathrm{kV}$. TEM observations were performed on a JEOL 2000 EX-II instrument operating at $160 \mathrm{keV}$. Suspensions of GO and TRGs were deposited on standard holey carbon copper grids using the drop cast method and loaded into the microscope. HR-TEM was performed on GO deposited on lacey carbon copper grids using drop cast and loaded into the microscope. Analyses were carried on a TITAN Cube microscope with a Cs-corrector in the objective lens to achieve resolutions below $0.1 \mathrm{~nm}$ in HRTEM mode. The microscope was operated at $80 \mathrm{keV}$. Raman spectroscopy was performed on a Renishaw 2000 Confocal Raman Microprobe (Rhenishaw Instruments, England) using a $514.5 \mathrm{~nm}$ argon ion laser. Spectra were recorded from 750 to $3500 \mathrm{~cm}^{-1}$. The size and height of the samples were measured by means of AFM imaging and profiling by depositing a drop of a water suspension of the samples onto the surface of mica. The sheets were imaged using a Cervantes atomic force microscope from Nanotec Electronica ${ }^{\mathrm{TM}}$ operating under ambient conditions. Microcantilevers with nominal spring constants of $\mathrm{k}=40 \mathrm{~N} / \mathrm{m}$ and a resonance frequency of $\mathrm{f}=300 \mathrm{kHz}$ were used to image the sheets. WSxM software was employed to control the atomic force microscope as well as for the data processing of the acquired images.

\section{Results and discussion}

The parent graphite was fully oxidized after 3 hours, as confirmed by XRD (Figure 1). Graphite has an intense crystalline peak at $26.5^{\circ}$ corresponding to the (002) plane; on the conversion to GO, the (002) and (101) peaks of graphite disappear while the (001) becomes 
evident at $2 \Theta=9.8^{\circ}$. The interlayer distance increases from $0.336 \mathrm{~nm}$ for the graphite to $0.867 \mathrm{~nm}$ for the GO, as a result of the expansion caused by the incorporation of water and oxygen functional groups during the oxidation process (Table 1). The oxygen content of the GO (Table 2 ) is very high ( $46.8 \%$ as determined by elemental analysis; $\mathrm{C} / \mathrm{O}$ ratio of 2.1 , as determined by XPS). This oxygen is mostly in the form of hydroxyl (26.4 \%), followed by epoxy (17.5\%) and carboxyl groups (11.9\%), according to XPS (Table 2). The presence of the last two groups was confirmed by FTIR (see S.I.). The epoxy and hydroxyl groups can be expected to be mainly located at the basal planes, in the interior of the aromatic domains, while the carboxyl groups will be located at the edges of the sheets or at the edges of holes, if at all present.

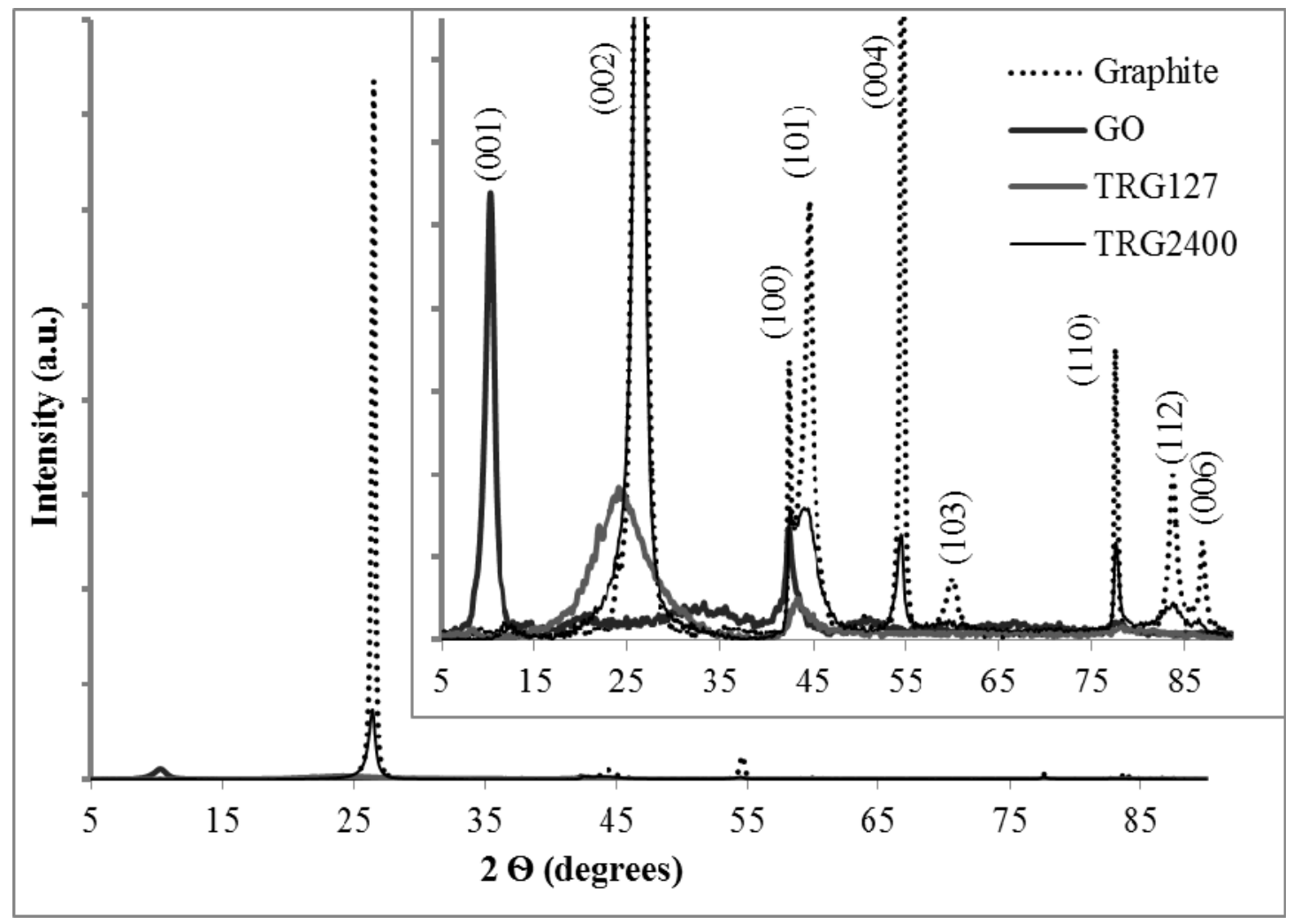

Figure 1. X-ray pattern for the graphite, the GO and the GO treated at $127{ }^{\circ} \mathrm{C}$ (TRG127) and at $2400^{\circ} \mathrm{C}$ (TRG2400). 
Single folded sheets of GO were observed after dispersion in water by TEM and confirmed by AFM, which shows the dominant presence of "monolayers" with an average height of $0.90 \mathrm{~nm}$ (see S.I.). The main structural features of the GO sheets, as evidenced by HRTEM (Figure 2) [14]: i) small domains of defect-free graphene (green colour), ii) large regions of well-structured graphene but with some $\mathrm{O} / \mathrm{OH}$ and some structural defects (blue colour), thus confirming the preferential location of these oxygen functional groups in the interior of the aromatic domains of the basal planes; and iii) disordered or amorphous graphene (yellow colour).

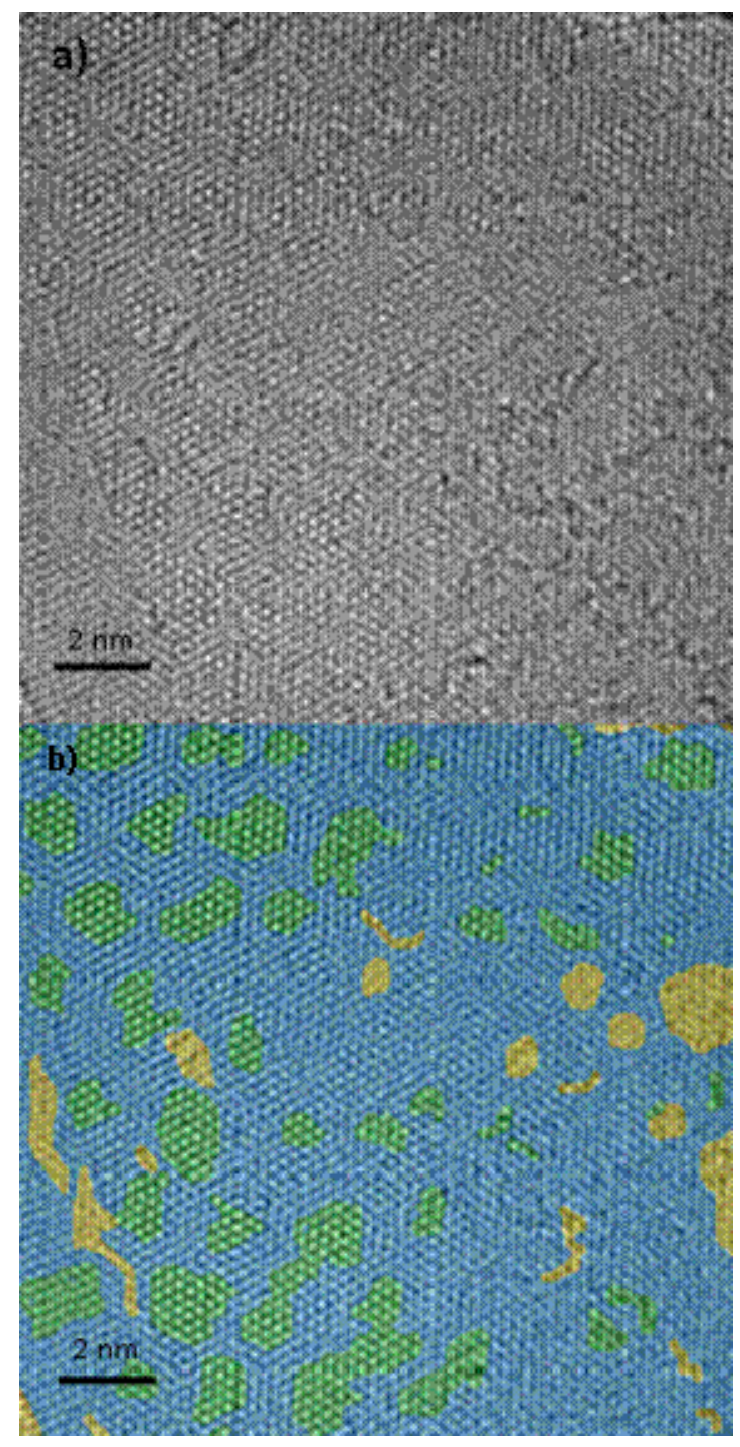


Figure 2. Aberration-corrected low energy $(80 \mathrm{keV})$ HRTEM images of water dispersed GO (a) and colored and filtered image (b). The following colors are used to represent the main structural features: green, ordered graphene structure; blue, ordered graphene structure with some $\mathrm{O} / \mathrm{OH}$ or some structural defects and yellow, disordered or amorphous graphene.

The blasting of GO at the experimental conditions used occurred at about $127{ }^{\circ} \mathrm{C}$, as evidenced by the sudden loss of $\mathrm{H}_{2} \mathrm{O}, \mathrm{CO}$ and $\mathrm{CO}_{2}$ detected by TPD (Figure 3 (a)). This temperature is slightly lower than those reported by other authors [15], probably as a consequence of the differences in the characteristics of the GOs (obtained from different graphites), since we also observed blasting temperatures of $\sim 200{ }^{\circ} \mathrm{C}$ when graphite oxide obtained from flake graphite was used. The blasting temperature of $127^{\circ} \mathrm{C}$ was the first temperature selected for the exfoliation/reduction of GO. The other temperatures used in this study were selected from the thermogravimetric analysis (TGA/DTG) of the graphite oxide exfoliated by sonication (Figure 3b). GO was not used directly for these experiments to avoid the blasting and blowing of the material out of the vessel. The TGA/DTG curves show that a small amount of water (3\%) was released during the initial heating stage (Figure 3 (b)), followed by a dramatic mass loss at $150-300{ }^{\circ} \mathrm{C}(15 \%)$, corresponding to the decomposition of oxygen functional groups. The products of this decomposition were found to be mainly $\mathrm{H}_{2} \mathrm{O}$ and $\mathrm{CO}_{2}$. There is a continuous and smooth weight loss (around 22 $\%$ ) in the temperature range of $350-1000{ }^{\circ} \mathrm{C}$ that corresponds to the loss of $\mathrm{CO}$ and $\mathrm{H}_{2}$. The mass loss during thermal decomposition was approximately $40 \%$. From these results the temperatures of $127{ }^{\circ} \mathrm{C}, 300{ }^{\circ} \mathrm{C}, 400{ }^{\circ} \mathrm{C}, 600{ }^{\circ} \mathrm{C}, 700{ }^{\circ} \mathrm{C}$ and $1000{ }^{\circ} \mathrm{C}$ were selected to perform the thermal exfoliation/reduction of GO, using a slow heating rate $\left(5^{\circ} \mathrm{C} \mathrm{min}^{-1}\right)$ to minimize the distortion of the sheets, but fast enough to propitiate the blasting, since the 
experiments carried out at $30{ }^{\circ} \mathrm{C} \mathrm{min}-1$ produced more wrinkled sheets and the exfoliation was lower. The temperatures of $2000{ }^{\circ} \mathrm{C}$ and $2400{ }^{\circ} \mathrm{C}$ were also used for the sake of completeness. The resultant samples were labelled TRGt, where $t$ indicates the temperature used. 


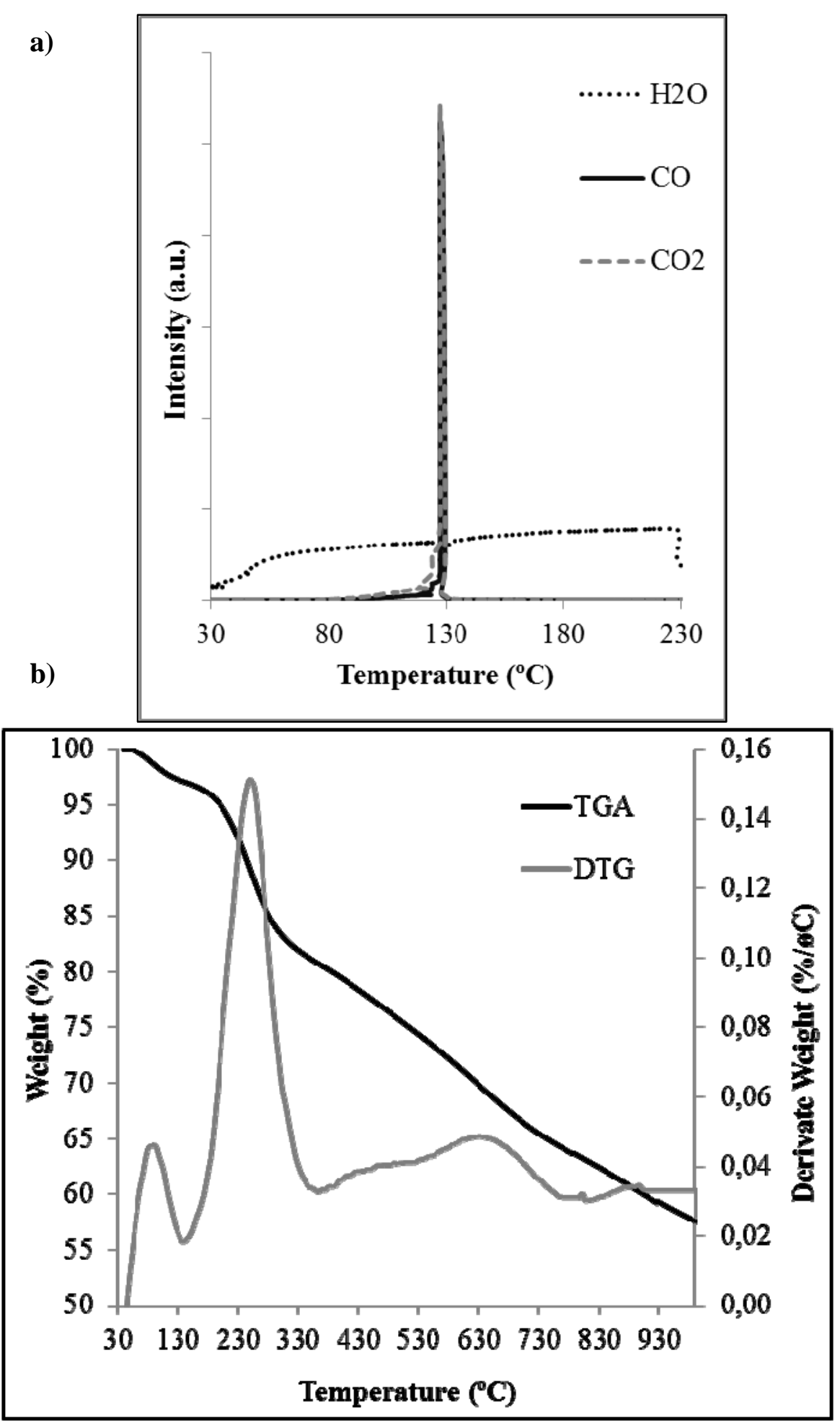

Figure 3. (a) TPD profile of GO showing blasting at $127^{\circ} \mathrm{C}$, (b) TGA profile of GO after exfoliation by ultrasonication. 
The as-prepared powder of the different samples had a typical agglomerated fluffy appearance as observed by SEM (Figure 4), as a result of the decomposition of the oxygen functional groups that leads to graphene-like sheets with a disordered stacking. No significant differences between the different TRGs were observed by SEM. The TEM images of the TRGs (Figure 5), after being dispersed in water/DMF (1:1), show that the wrinkled appearance of the sheets -typical of GOs when they are subjected to thermal treatment- becomes smoother at temperatures of $2000{ }^{\circ} \mathrm{C}$ and $2400{ }^{\circ} \mathrm{C}$.

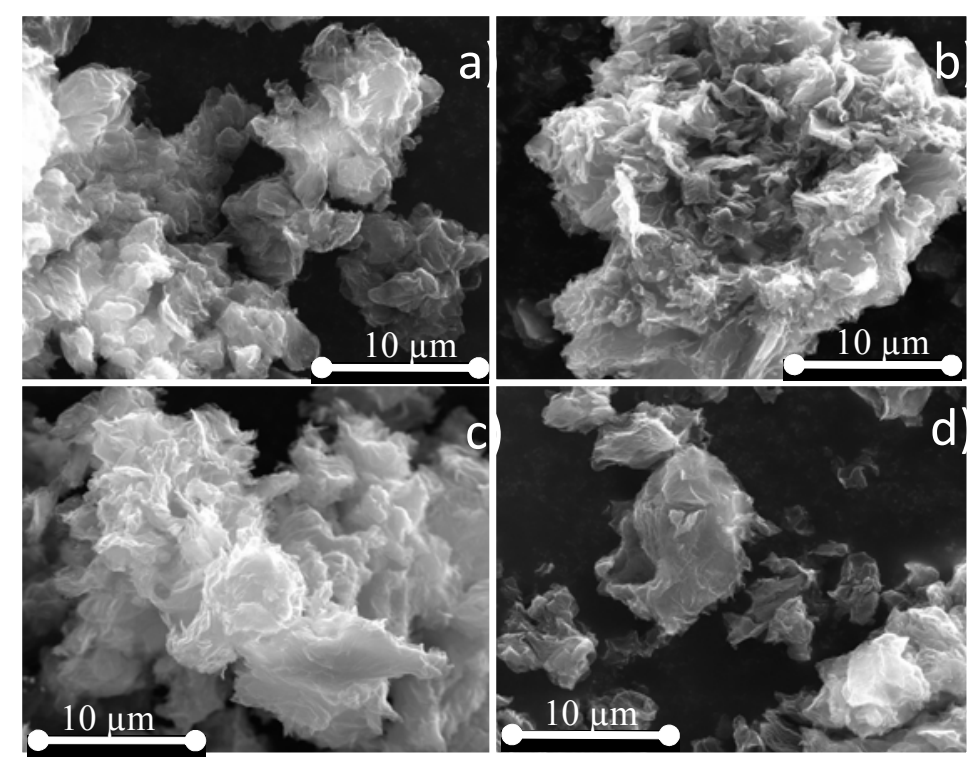

Figure 4. SEM image of powder TRGs obtained at: (a) $600{ }^{\circ} \mathrm{C}$, (b) $800{ }^{\circ} \mathrm{C}$, (c) $1000{ }^{\circ} \mathrm{C}$ and (d) $2000{ }^{\circ} \mathrm{C}$. 

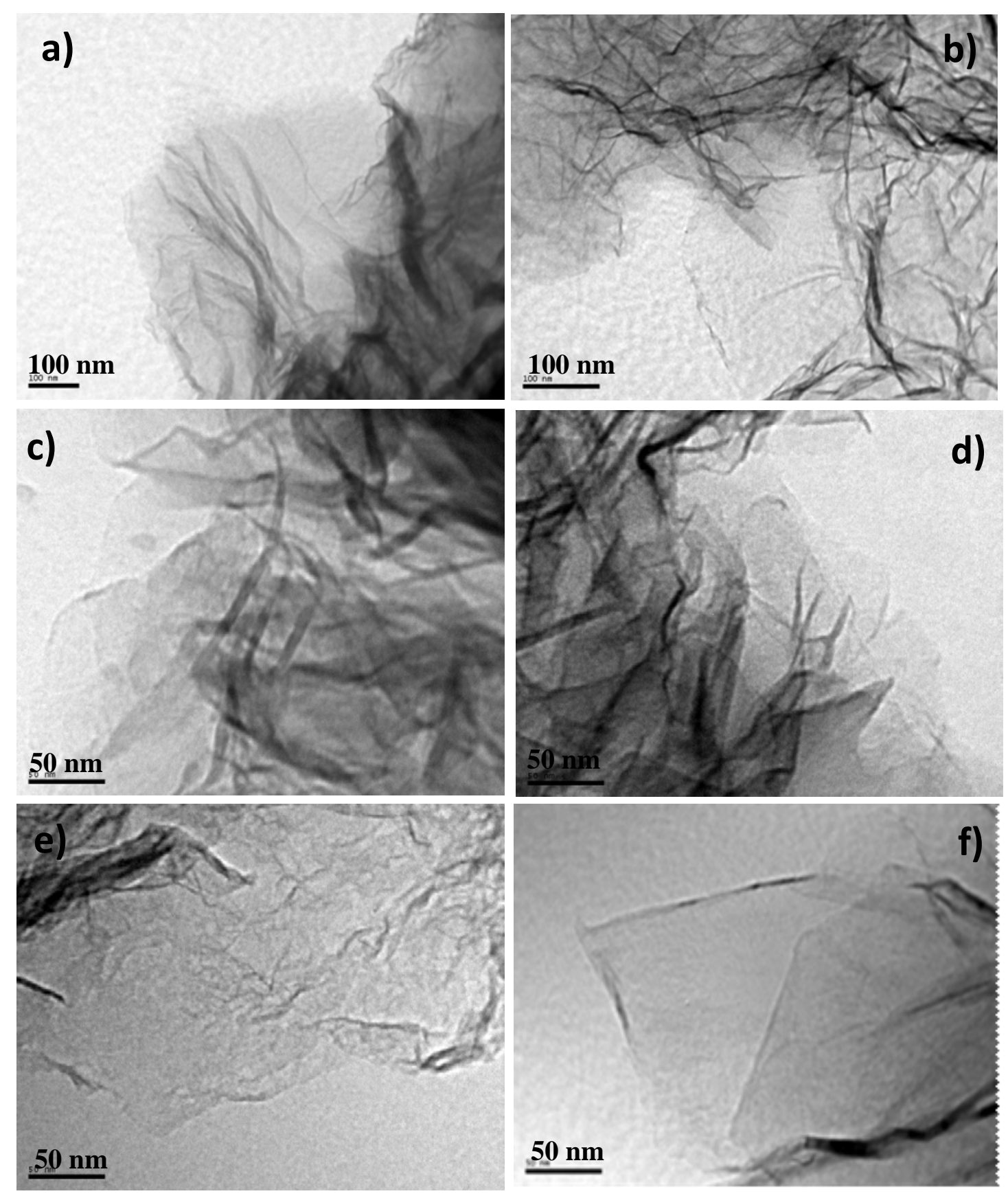

Figure 5. TEM images of the TRGs obtained at: (a) and (b) $127{ }^{\circ} \mathrm{C}$; (c) $400{ }^{\circ} \mathrm{C}$, (d) $800^{\circ} \mathrm{C}$, (e) $1000{ }^{\circ} \mathrm{C}$ and (f) $2000{ }^{\circ} \mathrm{C}$. Images of the TRG obtained at $2400{ }^{\circ} \mathrm{C}$ are similar to those of the $2000{ }^{\circ} \mathrm{C}$ one. TRGs are dispersed in water/DMF.

The change in colour from brown in the GO to black in TRG127 (Figure 6) evidences that thermal reduction starts at this very low temperature, when blasting occurs as a 
consequence of the sudden release of gases, producing a significant increase in volume (see S.I.), accompanied by the exfoliation and reduction of GO, with a significant loss of oxygen. TEM images of TRG127 showing the typical morphology of graphene sheets with a wrinkled structure (Figure $5 \mathrm{a}$ and $5 \mathrm{~b}$ ) are indicative of these phenomena.

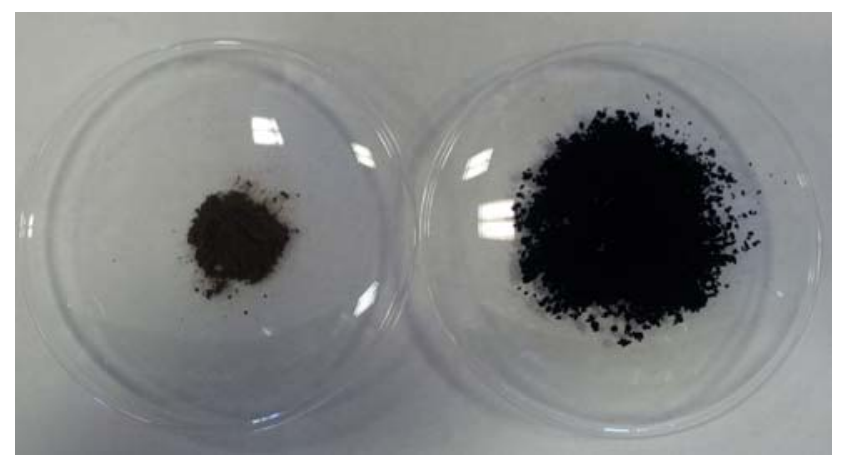

Figure 6. Digital images showing the increase in volume after blasting and the colour change from brown to black as a consequence of the thermal reduction. GO (left) and TRG127 (right).

The successful exfoliation of TRG127 is reconfirmed by means of the analysis of the XRD pattern obtained for the samples in the powder form (Figure 1), by the appearance of the (002) peak at $26^{\circ}$ and the disappearance of the GO (001) peaks and the corresponding increase in d-spacing (from $0.336 \mathrm{~nm}$ from graphite to $0.366 \mathrm{~nm}$, Table 1). The XRD pattern shows a broad peak for TRG127 situated at a value of $2 \Theta$ slightly lower than for graphite. There is a progressive narrowing of the peak with increasing temperature of treatment and a subsequent shift towards the d-spacing of graphite (Table 1). In TRG2000 and TRG2400 the peak (101) becomes evident although the pattern indicates that these are heavily disordered carbons [10]. The d-spacing almost has the same value than that of the 
parent graphite. The $\mathrm{L}_{\mathrm{c}}$ values of TRG2000 and TRG2400, $11 \mathrm{~nm}$ and $15 \mathrm{~nm}$, respectively, are substantially lower than that of the initial graphite $(37 \mathrm{~nm})$. This suggests that, in the powder form, the graphene sheets obtained after blasting do associate progressively during the thermal treatment but not at the level typical for crystalline graphite. The number of layers, roughly estimated by the equation $(\mathrm{Lc} / \mathrm{d})+1$, increases from 5 at $127^{\circ} \mathrm{C}$ to 9 at 800 ${ }^{\circ} \mathrm{C}$, and more drastically above this temperature (18 at $1000{ }^{\circ} \mathrm{C}$ and 45 at $2400{ }^{\circ} \mathrm{C}$ ). This proves two important points: i) an excellent exfoliation of the graphite is achieved at 127 ${ }^{\circ} \mathrm{C}$ and ii) temperatures above $1000{ }^{\circ} \mathrm{C}$ favour the stacking of the sheets (more than 10 ), although the crystalline graphite status is not reached even at $2400{ }^{\circ} \mathrm{C}$. This is confirmed by the fact that TRG1000, TRG2000 and TRG2400 can be delaminated and dispersed in appropriate solvents by sonication, something that is not possible with crystalline graphite. A possible explanation can be found in their disordered and disrupted structure that greatly reduces the attractive interactions between the layers, allowing this material to be easily dispersed in solvents, as stated by McAllister et al. [10].

AFM is the most direct method of quantifying the degree of exfoliation to a single graphene sheet level after the dispersion of the powder in a solvent. The height of the TRGs sheets dispersed in water/DMF shows the dominant presence of monolayers at $127^{\circ} \mathrm{C}$ and $300{ }^{\circ} \mathrm{C}, 1-2$ layers at $400{ }^{\circ} \mathrm{C}, 2-4$ layers at $600{ }^{\circ} \mathrm{C}, 3-4$ layers at $800{ }^{\circ} \mathrm{C}, 4-5$ layers at 1000 ${ }^{\circ} \mathrm{C}$ and 4-6 layers at $2000{ }^{\circ} \mathrm{C}$ and $2400{ }^{\circ} \mathrm{C}$ (Table 1). Figure 7 shows $\mathrm{AFM}$ images and profiles corresponding to TRG300, TRG600, TRG1000 and TRG2400. Our AFM results support the above discussion on the XRD results concerning the number of layers (Figure 7) and confirm that even at temperatures above $2000^{\circ} \mathrm{C}$ few-layer graphenes can be obtained after TRGs dispersion in water/DMF. 
Table 1 XRD analysis of samples in the powder form: interlaminar distance, crystallite size and estimated number of layers and number of layers as determined by AFM on the dispersed samples.

\begin{tabular}{cccccc}
\hline & & XRD & & \multicolumn{2}{c}{ AMF } \\
\hline & $\mathbf{d}_{\mathbf{0 0 2}}(\mathrm{nm})^{\mathrm{a}}$ & $\mathbf{L}_{\mathbf{c}}(\mathrm{nm})^{\mathrm{b}}$ & $\mathbf{n}^{\mathrm{c}}$ & $\mathbf{h ~ ( n m ) ~}^{\mathbf{d}}$ & $\mathbf{n}^{\mathrm{e}}$ \\
\hline Graphite & 0.336 & 36.98 & 111 & ---- & - \\
TRG127 & 0.366 & 1.43 & 5 & 1.2 & 1 \\
TRG300 & 0.365 & 1.36 & 5 & 0.8 & 1 \\
TRG400 & 0.356 & 2.06 & 7 & 2.2 & $1-2$ \\
TRG600 & 0.347 & 2.11 & 7 & 2.2 & $2-4$ \\
TRG700 & 0.345 & 2.35 & 8 & 3.0 & $2-4$ \\
TRG800 & 0.343 & 2.63 & 9 & 2.6 & $3-4$ \\
TRG1000 & 0.340 & 5.67 & 18 & 5.0 & $4-5$ \\
TRG2000 & 0.338 & 10.50 & 32 & 4.5 & $4-6$ \\
TRG2400 & 0.338 & 14.94 & 45 & 5.5 & $4-6$ \\
\hline
\end{tabular}

${ }^{a}$ interlaminar distance; ${ }^{b}$ crystallite size; ${ }^{c}$ number of layers estimated by XRD from $\left(\mathrm{L}_{\mathrm{c}} / \mathrm{d}_{002}\right)+1 ;{ }^{\mathrm{d}}$ average height of events determined by $\mathrm{AFM}{ }^{\mathrm{e}}$ number of layers determined by AFM. 

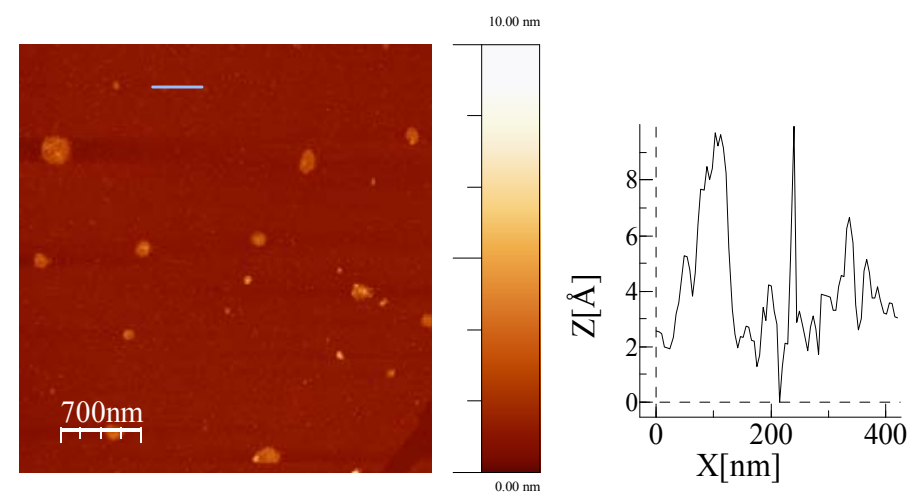

a)TRG30
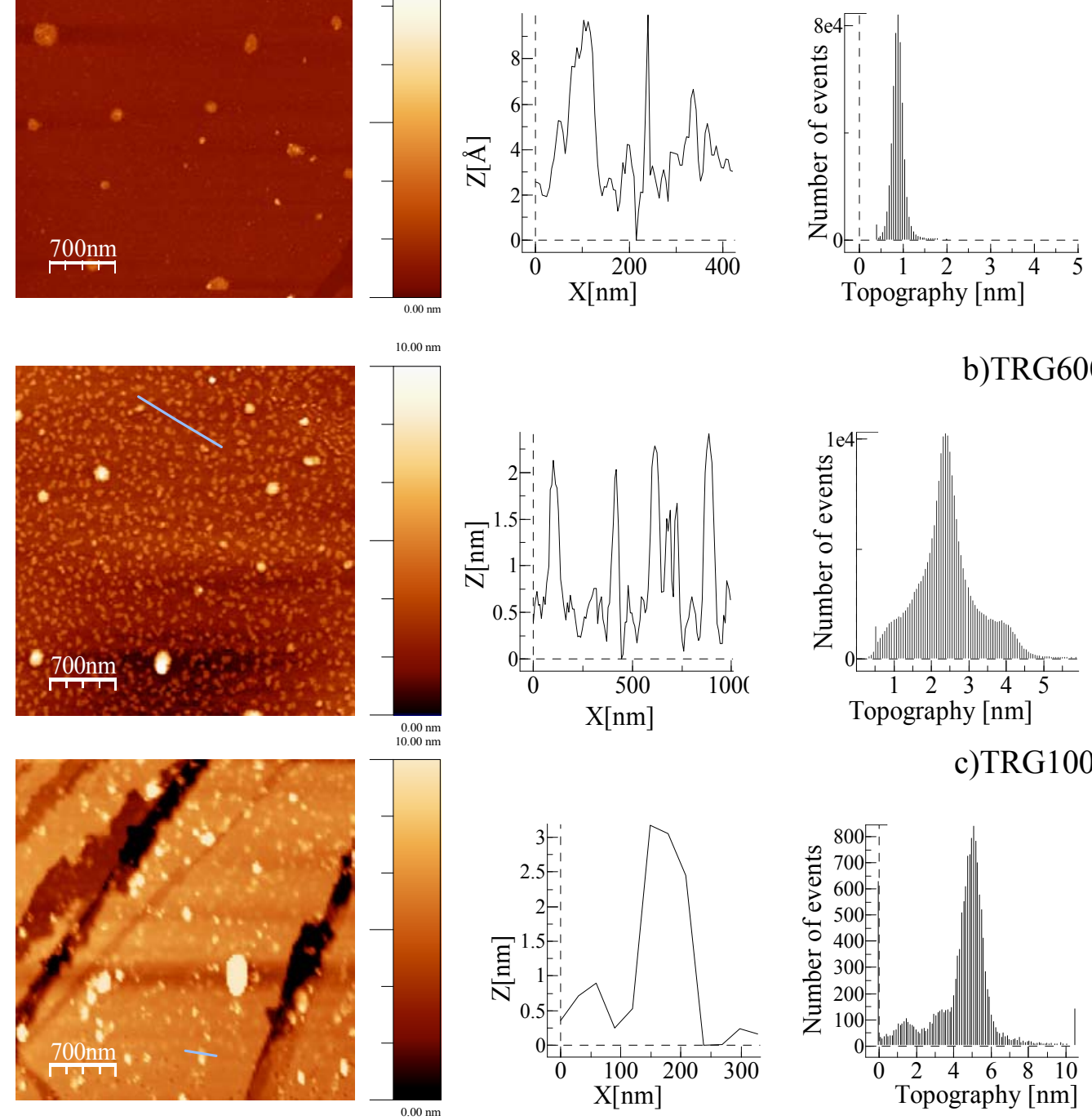

b)TRG600

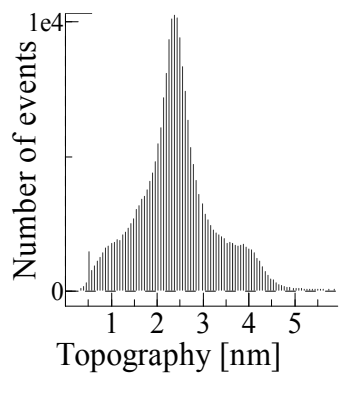

c)TRG100
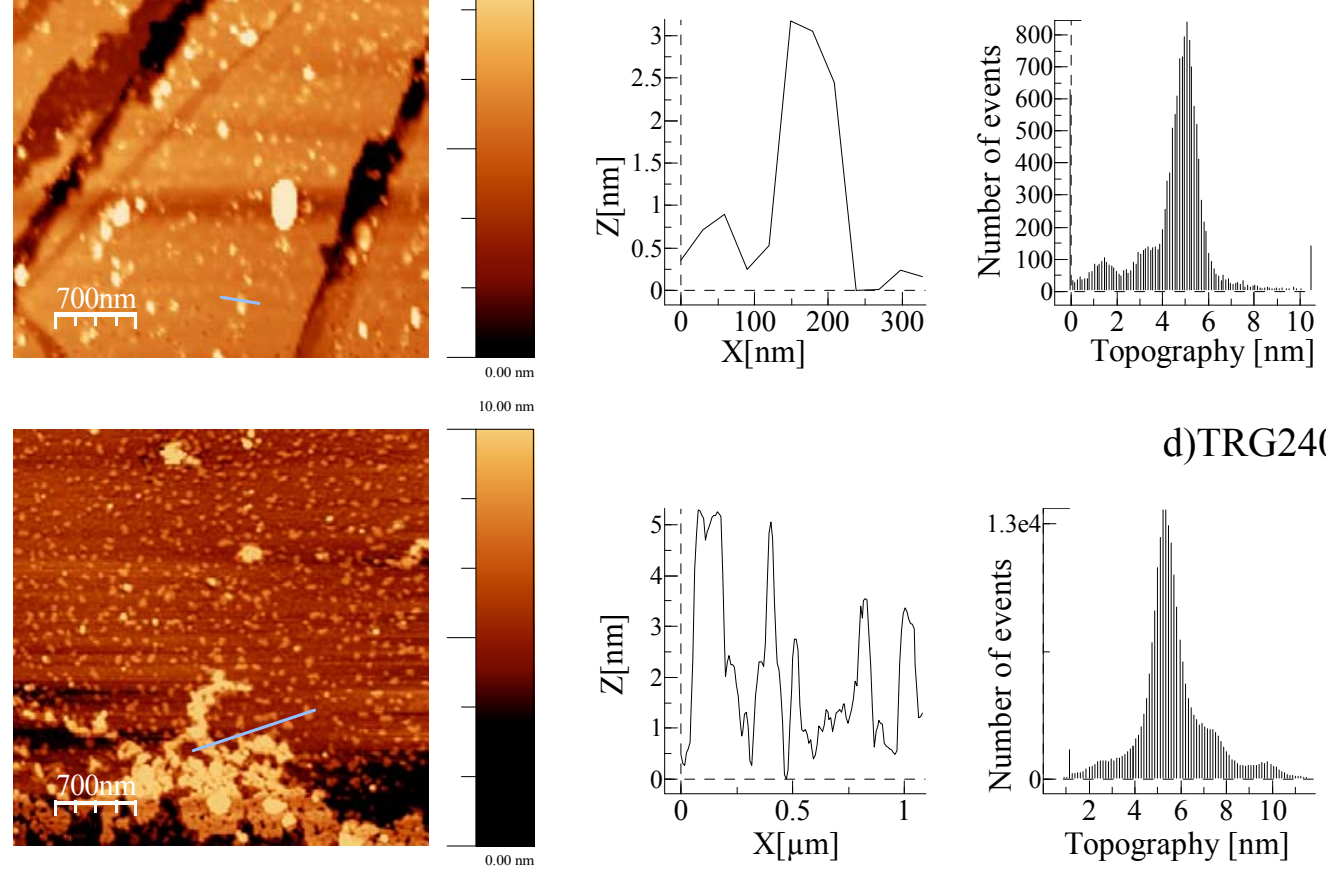

d)TRG2400

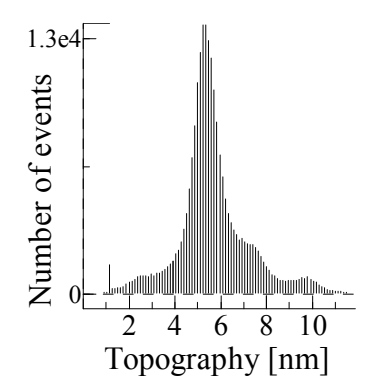

Figure 7. AFM images of TRGs sheets (a) TRG300, (b) TRG600, (c) TRG1000 and (d) TRG2400. The horizontal lines indicate the sections corresponding to the traces shown on the right. The histograms show the height distributions of the sheets analyzed. 
The increasing tendency to associate with increasing temperature may be the result of the increasing absence of oxygen functional groups. The main oxygen loss $(25 \%)$ occurs at $127{ }^{\circ} \mathrm{C}$, the oxygen content decreasing from $47 \%$ in the GO to $22 \%$ in TRG127 (as determined by elemental analysis, Table 2). According to Gao et al. [3], this loss mainly corresponds to hydroxyl and epoxy groups located in the interior of the aromatic domains and carboxylic groups located at the edges (as confirmed by XPS, Table 2), as they are less stable, decreasing from $26 \%, 17 \%$ and $12 \%$ (hydroxyl/epoxy/carboxyl) for GO to $9 \%, 6$ $\%$ and $4 \%$ after the heat treatment at $127{ }^{\circ} \mathrm{C}$. The restoration of the $\mathrm{Csp}^{2}$ structure is also noticeable at $127{ }^{\circ} \mathrm{C}$, increasing from $36 \%$ in the GO to $69 \%$ in TRG127. The slight increase in the amount of $\mathrm{Csp}^{3}$ (from $9 \%$ to $13 \%$ ) is a consequence of the distortion of the carbon lattice during de-oxygenation. No significant variation is observed from 127 to 300 ${ }^{\circ} \mathrm{C}$. At $600{ }^{\circ} \mathrm{C}$ the $\mathrm{Csp}^{2}$ continues to increase up to $73 \%$, mainly due to the removal of the remaining hydroxyl and epoxy groups. It was observed that the removal of these groups also contributes to distort the carbon lattice (increment of $\mathrm{Csp}^{3}$ up to $17 \%$ ). At $700{ }^{\circ} \mathrm{C}$ and $800{ }^{\circ} \mathrm{C}$ the changes are very small and there is again a more marked removal of oxygen at $1000{ }^{\circ} \mathrm{C}$, its content decreasing to about $2 \%$. At the same time, a noticeable increase in the $\mathrm{Csp}^{2}$ lattice is obtained (up to $82 \%$ ), this meaning that the thermal reduction has been very effective. Oxygen functional groups almost disappear at 2000 and $2400{ }^{\circ} \mathrm{C}$ and the main features observed are the conversion of the $\mathrm{Csp}^{3}$ lattice to form the typical $\mathrm{Csp}^{2}$ graphene lattice, with values up to $86 \%$. 
Table 2. Fitted results of the C1s, O1s and N1s core level XPS spectra and the oxygen content of the GO and TRGs.

\begin{tabular}{cccccccc}
\hline & $\begin{array}{c}\mathbf{O}^{\mathbf{a}} \\
(\mathbf{\%})\end{array}$ & C/O & $\begin{array}{c}\mathbf{C s p}^{2} \\
(\%)\end{array}$ & $\begin{array}{c}\mathbf{C s p}^{3} \\
(\mathbf{\%})\end{array}$ & $\begin{array}{c}\text { C-OH } \\
(\%)\end{array}$ & $\begin{array}{c}\text { O-C-O } \\
(\%)\end{array}$ & $\begin{array}{c}\text { OH-C=O } \\
(\%)\end{array}$ \\
\hline GO & 46.8 & 2.3 & 36.2 & 9.3 & 26.4 & 17.5 & 11.9 \\
TRG127 & 21.8 & 5.3 & 61.3 & 19.5 & 8.8 & 6.4 & 4.1 \\
TRG300 & 22.8 & 5.3 & 68.7 & 13.2 & 8.7 & 5.7 & 3.7 \\
TRG400 & 18.9 & 5.8 & 70.3 & 15.2 & 7.1 & 4.8 & 2.6 \\
TRG600 & 11.3 & 10.7 & 73.1 & 17.2 & 4.6 & 3.0 & 2.1 \\
TRG700 & 13.0 & 8.5 & 74.0 & 17.7 & 4.4 & 2.5 & 1.4 \\
TRG800 & 5.5 & 9.4 & 75.5 & 18.2 & 3.3 & 2.0 & 1.0 \\
TRG1000 & 1.9 & 10.7 & 81.5 & 15.2 & 2.1 & 1.1 & 0.1 \\
TRG2000 & 0.2 & 35.1 & 83.2 & 15.4 & 1.4 & 0.0 & 0.0 \\
TRG2400 & 0.1 & 39.8 & 85.8 & 13.6 & 0.6 & 0.0 & 0.0 \\
\hline
\end{tabular}

${ }^{\text {a }}$ Determined by elemental analysis

The significant structural changes occurring from graphite to GO and then to TRGs are also reflected in the results obtained by Raman spectroscopy (Figure 8 and S.I.). The Raman spectrum of the graphite exhibits a characteristic strong $G$ band at $1583 \mathrm{~cm}^{-1}$, which is attributed to the vibration of $\mathrm{sp}^{2}$-bonded atoms in a two-dimensional hexagonal lattice, as well as a weak D band at $1365 \mathrm{~cm}^{-1}$, which is caused by carbon atoms at the layer edges or imperfections $\left(\mathrm{I}_{\mathrm{D}} / \mathrm{I}_{\mathrm{G}}\right.$ ratio 0.12$)$. In the Raman spectrum of $\mathrm{GO}$, the $\mathrm{G}$ band is broadened and the $\mathrm{D}$ band becomes prominent $\left(\mathrm{I}_{\mathrm{D}} / \mathrm{I}_{\mathrm{G}}\right.$ ratio 0.91$)$, indicating the reduction in size of the $\mathrm{sp}^{2}$ domains due to the extensive oxidation. The disorder increases for the samples obtained at temperatures to $1000{ }^{\circ} \mathrm{C}\left(\mathrm{I}_{\mathrm{D}} / \mathrm{I}_{\mathrm{G}}\right.$ ratio 1.17$)$. This change suggests a decrease in the 
average size of the $\mathrm{sp}^{2}$ domains upon reduction of the exfoliated GO and can be explained if new graphitic domains are smaller in size than those present in GO before reduction, but more numerous. Samples obtained at 2000 and $2400{ }^{\circ} \mathrm{C}$ show $\mathrm{I}_{\mathrm{D}} / \mathrm{I}_{\mathrm{G}}$ ratios close to that of the parent graphite ( 0.15 and 0.14 respectively), indicating that it is at these temperatures that we have a real restoration of the graphite lattice in the layers. A schematic model of the main features of the TRGs obtained at the different temperatures is shown in Figure 9.

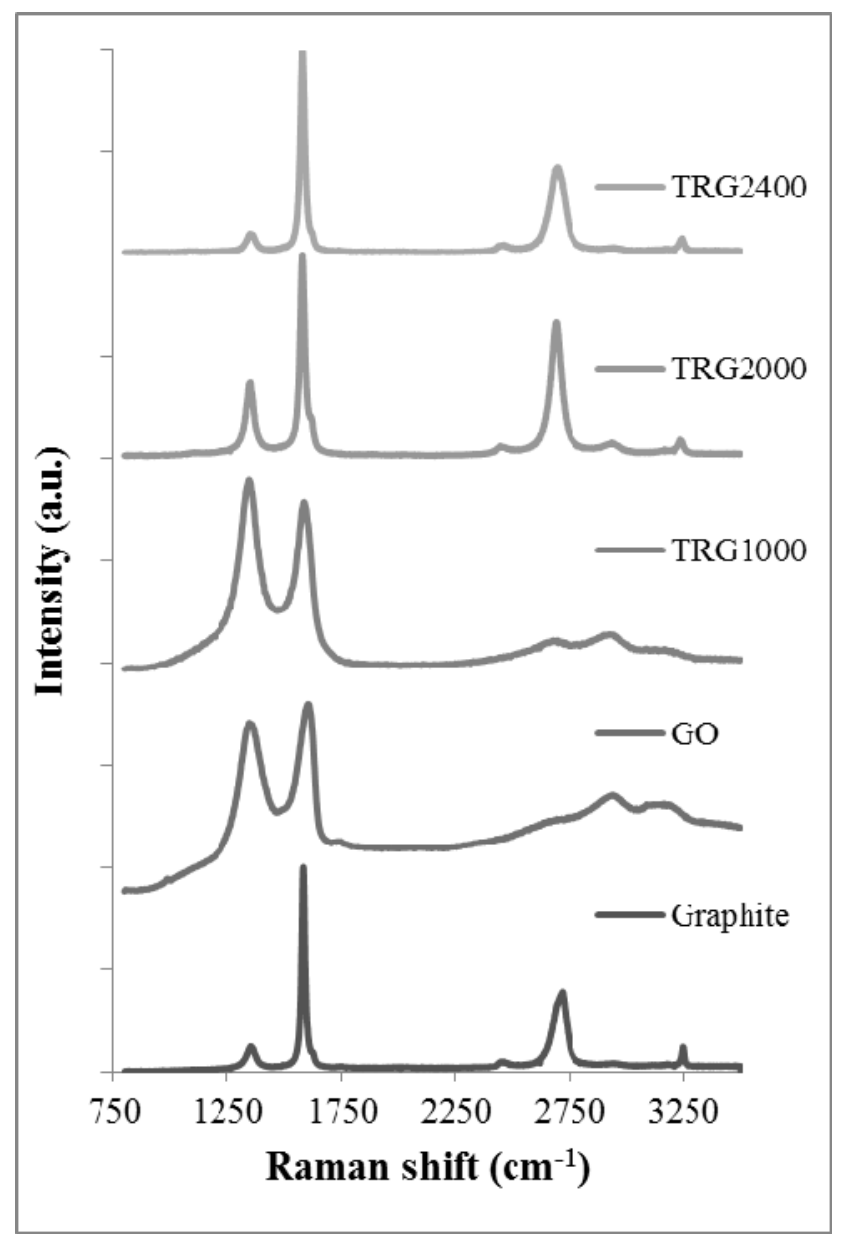

Figure 8. Raman spectra of parent graphite, GO, TRG1000, TRG2000 and TRG2400. 


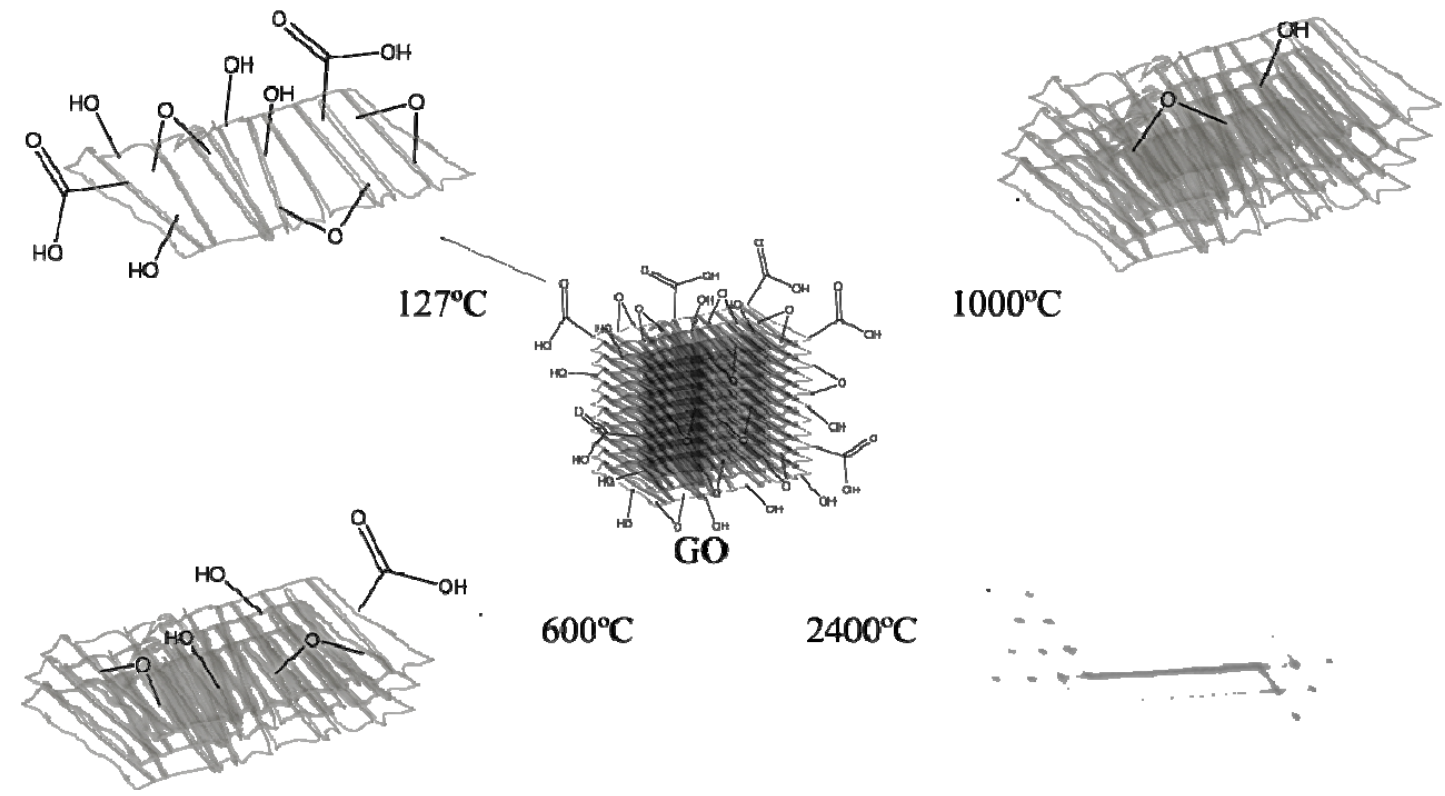

Figure 9. Schematic model of the main features of the TRGs obtained at the different temperatures.

\section{Conclusions}

Exfoliation of synthetic graphite powder oxide takes place at a temperature as low as 127 ${ }^{\circ} \mathrm{C}$, producing functionalized monolayer graphene when dispersed in water/DMF. The product contains $22 \%$ oxygen, mainly in the form of hydroxyl groups and minor amounts of epoxy and carboxyl groups. The exfoliation also implies a partial reduction as a consequence of the substantial loss of oxygen surface groups. When the GO is treated at higher temperatures, especially above $600{ }^{\circ} \mathrm{C}$, the reduction improves, with a loss of oxygen and hydrogen and a conversion of hybridized carbon atoms from $\mathrm{sp}^{3}$ into $\mathrm{sp}^{2}$. The amount of carboxyl and epoxy groups decreases from 400 to $1000{ }^{\circ} \mathrm{C}$, the hydroxyl group content remaining almost constant. $1000^{\circ} \mathrm{C}$ appears to be a critical temperature in the $\mathrm{GO}$ 
treatment from the point of view of the efficiency of the reduction process, as the resulting graphene-like material contained less than $2 \%$ oxygen and $81.5 \% \mathrm{Csp}^{2}$. The materials obtained at $2000{ }^{\circ} \mathrm{C}$ and $2400{ }^{\circ} \mathrm{C}$ were almost completely oxygen-free (less than $1 \%$ ) and the layers exhibited a dramatic restoration of the pristine graphite structure, as confirmed by the increase in the average size of the $\mathrm{sp}^{2}$ domains determined by Raman spectroscopy. The typical disordered stacking of TRGs increases with temperature, although they can be dispersed by ultrasonication in appropriate solvents, yielding monolayers (in those obtained at 127 and $300^{\circ} \mathrm{C}$ ) and stacks of up to 4-6 layers at temperatures above $1000^{\circ} \mathrm{C}$, as determined by AFM.

ACKNOWLEDGMENT. The authors thank the Spanish Science and Innovation Ministry (CONSOLIDER INGENIO 2010 CSD2009-00050, MAT2010-16194) for their financial support. Dr. Patricia Alvarez thanks the Spanish Science and Innovation Ministry for her Ramon y Cajal contract. Cristina Botas acknowledges a fellowship from FICYT. This paper is dedicated to our friend Prof. Puri Escribano (in memoriam). 


\section{REFERENCES}

[1] Park S, Ruoff, RS. Chemical methods for the production of graphenes. Nat Nanotechnol $2009 ; 4: 217-24$.

[2] Marcano DC, Kosynkin DV, Berlin JM, Sinitskii A, Sun Z, Slesarev A, et al. Improved synthesis of graphene oxide. ACS Nano 2010; 4: 4806-14.

[3] Gao X, Jang J, Nagase S. Hydrazine and thermal reduction of graphene oxide: Reaction mechanisms, product structures, and reaction design. J Phys Chem C 2010; 114: 832-42.

[4] Botas C, Álvarez P, Blanco C, Santamaría R, Granda M, Ares P, et al. The effect of the parent graphite on the structure of graphene oxide. Carbon 2012; 50: 275-82.

[5] Stankovich S, Dikin DA, Piner RD, Kohlhaas KA, Kleinhammes A, Jia Y, et al. Synthesis of graphene-based nanosheets via chemical reduction of exfoliated graphite oxide. Carbon 2007; 45: 1558-65.

[6] Kaniyoor A, Baby TT, Arockiadoss T, Rajalakshmi N, Ramaprabhu S. Wrinkled graphenes: A study on the effects of synthesis parameters on exfoliation-reduction of graphite oxide. J Phys Chem C 2011; 115: 17660-9.

[7] Sundaram RS, Gomez-Navarro C, Balasubramanian K, Burghard M, Kern K. Electrochemical modification of graphene. Adv Mater 2008; 20: 3050-3.

[8] González Z, Botas C, Alvarez P, Roldán S, Blanco C, Santamaría R, Granda M, Menéndez R. Thermally reduced graphite oxide as positive electrode in Vanadium Redox Flow Batteries. Carbon 2012; 50: 828-34. 
[9] Schniepp HC, Li JL, McAllister MJ, Sai H, Herrera-Alonso M, Adamson DH, et al. Functionalized Single Graphene Sheets Derived from Splitting Graphite Oxide. Phys Chem C 2006; 110: 8535-9.

[10] McAllister MJ, Li JL, Adamson DH, Schniepp HC, Abdala AA, Liu J, et al. Single sheet functionalized graphene by oxidation and thermal expansion of graphite. Chem Mater 2007; 19: 4396-404.

[11] Bagri A, Mattevi C, Acik M, Chabal Y, Chhowalla M, Shenoy V. Structural evolution during the reduction of chemically derived graphene oxide. Nat Chem 2010; 2: 581-7.

[12] Hummers WS, Offeman RE. Preparation of Graphitic Oxide. J Am Chem Soc 1958; 80: $1339-40$.

[13] Yang D, Velamakanni A, Bozoklu G, Park S, Stoller M, Piner RD, et al. Chemical analysis of graphene oxide films after heat and chemical treatments by X-ray photoelectron and micro-Raman spectroscopy. Carbon 2009; 47: 145-52.

[14] Botas C, Álvarez P, Blanco C, Gutiérrez MD, Ares P, Zamani R, et al. Tailored graphene materials by chemical reduction of graphene oxides of different atomic structure. RSC Adv DOI: 10.1039/c2ra21447d.

[15] Boehm HP, Scholz W. Der "verpuffungspunkt" des graphitoxids. Anorg Allg Chem 1965; 335: 74-9. 